Pacific Journal of Mathematics

A CHARACTERIZATION OF RESTRICTIONS OF
FOURIER-STIELTJES TRANSFORMS 


\title{
A CHARACTERIZATION OF RESTRICTIONS OF FOURIER-STIELTJES TRANSFORMS
}

\author{
HASKell P. Rosenthal
}

The main result that we prove here is as follows: Let $E$ be a Lebesgue measurable subset of $R$, the real line, and let $\varphi$ be a bounded measurable function defined on $E$. Then the first of the following conditions implies the second:

(1) There exists a constant $K$, so that

$$
\left|\sum_{j=1}^{n} c_{j} \varphi\left(x_{j}\right)\right| \leqq K\|P\|_{\infty}
$$

for all trigonometric polynomials of the form

$$
P(y)=\sum_{j=1}^{n} c_{j} e^{i x} j^{y}, \text { where } x_{j} \in E \text { for all } 1 \leqq j \leqq n .
$$

(2) $\varphi$ is $E$-almost everywhere a Stieltjes transform. Precisely, there exists a finite (complex Borel) measure $\mu$, so that

$$
\varphi(x)=\hat{\mu}(x)=\int_{-\infty}^{\infty} e^{-i x y} d \mu(y)
$$

for almost all $x \in E$. Moreover, $\mu$ may be chosen such that $\|\mu\| \leqq K$, where $K$ is the constant in (1). ( $\mid \mu \|$ denotes the total variation of $\mu$.)

In 1934 (c.f. [3]), Bochner established this result for the case when $E$ is the entire real line. Our result also generalizes a theorem of Krein. Indeed Krein proved (c.f. [1] pp. 154-159) that (1) and (2) are equivalent for the case when $E$ is an interval and $\varphi$ is a continuous function defined on $E$. Now if we assume that $E$ is closed and of uniformly positive measure, (meaning that if $U$ is an open subset of $\boldsymbol{R}$ with $U \cap E$ nonempty, then the measure of $U \cap E$ is positive), and if $\varphi \in C(E)$ and satisfies (1), then our result implies that (2) holds for all $x \in E$. (i.e. $\varphi \equiv \hat{\mu} \mid E$ for some finite measure $\mu$ on $\boldsymbol{R}$ ). (It is trivial that (2) implies (1) under these hypotheses.)

Note finally that it $E$ is a closed subset of $T$, the circle group, of uniformly positive measure, and if $\varphi \in C(E)$ and satisfies (2), then $\varphi \in A(E)$. That is, $\varphi$ can be extended to a function defined on all of $\boldsymbol{T}$, with absolutely convergent Fourier series. (We identify $\boldsymbol{T}$ with the real numbers modulo 1 ; in this case, the polynomials of condition (2) are almost-periodic functions defined on the integers.)

We obtain our main result by first proving the result mentioned in the above paragraph in Theorem 3; next by establishing the analogue of the main result for $\boldsymbol{T}$ in Theorem 4, and finally by passing from the circle to the real line in $\S 3$. 
The core of the proof of Theorem 3 is found in Lemma 2; the technique used there was suggested by a method due to C.S. Herz, as exposed in Théorème VII, pp. 124-126 of [4]. An essential step in the proof of Lemma 2 is Lemma 1, where we show that a measurable subset of $\boldsymbol{T}$ may be approximated in measure by nicely-placed closed subsets ${ }^{1}$.

1. Preliminaries. The following two results are not essential for the main result, but they do provide some motivation for it. We let $\boldsymbol{Z}$ denote the integers; if $\mu$ is a finite measure on $R$ (resp. $T$ ), $\|\hat{\mu}\|_{\infty}=\sup _{x \in R}|\hat{\mu}(x)|\left(\operatorname{resp} . \sup _{n \in R}|\hat{\mu}(n)|\right.$ where $\hat{\mu}(n)=\int_{0}^{1} e^{-i 2 \pi n t} d \mu(t)$ for all $n \in \boldsymbol{Z})$.

Proposition A. Let $E$ be an arbitrary subset of $\boldsymbol{R}$ (resp. $\boldsymbol{T}$ ), and let $\varphi$ be a bounded function defined on $E$. Then the following two conditions are each equivalent to (1).

(3) There exists a constant $K$, so that

$$
\left|\int \phi d \mu\right| \leqq K\|\hat{\mu}\|_{\infty}
$$

for all discrete measures $\mu$ supported on $E$.

(4) There exists a finite (complex regular Borel) measure $\nu$ defined on the Bohr compactification of $\boldsymbol{R}$ (resp. of $\boldsymbol{Z}$ ), so that $\varphi(x)=$ $\hat{\nu}(x)$ for all $x \in E$.

The fact that (1) and (3) are equivalent is a triviality. The equivalence of (1) and (4) is a consequence of the Riesz-representation theorem, together with the fact that the almost-periodic polynomials on $\boldsymbol{R}$ (resp. $\boldsymbol{Z}$ ) may be regarded as being dense in the space of continuous functions on the Bohr compactification of the respective groups. (See [5], pp. 30-32, for these and other properties of the Bohr compactification).

For the next proposition, we recall that for $E$ a closed subset of $\boldsymbol{T}, A(E)$ is the set of all $\phi \in C(E)$ for which there exists an $f \in \mathrm{C}(\boldsymbol{T})$, such that $f(x)=\varphi(x)$ for all $x \in E$, and $\sum_{n=-\infty}^{\infty}|\hat{f}(n)|<\infty . A(E)$ is. a Banach algebra under the norm

$$
\|\varphi\|_{\boldsymbol{A}(E)}=\inf \left\{\sum_{-\infty}^{\infty}|\hat{f}(n)|: f \in A(T) \text { with } f \mid E=\varphi\right\} .
$$

Proposition B. Let $E$ be a closed subset of $\boldsymbol{T}$ such that if

1 Benjamin Halpern independently discovered a different proof of Lemma 1, and we are indebted to him for a stimulating discussion concerning this result. 
$\varphi \in C(E)$ and $\varphi$ satisfies (3), then $\varphi \in A(E)$. Then there exists a finite constant $K$, so that for all $f \in A(E)$,

$$
\begin{gathered}
\|f\|_{A(E)} \leqq K\|\mid f\|, \text { where } \\
\|\| f \||=\sup | \int f d \mu \mid, \quad \text { the supremum }
\end{gathered}
$$

being taken over all discrete measures $\mu$ supported on $E$ with $\|\hat{\mu}\|_{\infty} \leqq 1$.

Proof. II $\cdot \mid \|$ defines a new norm on $A(E)$, and we have that \|\|$f\|\mid \leqq\| f \|_{A(E)}$, for all $f \in A(E)$. Now our hypotheses imply that $A(E)$ is complete under this norm also. Indeed, suppose $\left\{f_{n}\right\}$ is a Cauchy sequence in the norm $\|\cdot\| \|_{\text {. Fix }} x \in E$, and let $\mu_{x}$ be the measure assigning a mass of one to $x$. Then $\left\|\hat{\mu}_{x}\right\|_{\infty}=1$, so we have that

$$
\left\||| f_{n}-f_{m}\right\| \geqq \geqq\left|\int\left(f_{n}-f_{m}\right) d \mu_{x}\right|=\left|f_{n}(x)-f_{m}(x)\right|
$$

for all integers $n$ and $m$. Hence, $\left\{f_{n}\right\}$ is a Cauchy sequence in $C(E)$, so $\left\{f_{n}\right\}$ converges uniformly to a continuous function $\varphi$. Also, since $\left\{f_{n}\right\}$ is a Cauchy sequence in ||$\cdot \mid \|$, there exists a constant $K$ so that $\left\|\mid f_{n}\right\| \leqq K$ for all $n$. This means that

$$
\left|\int f_{n} d \mu\right| \leqq K\|\hat{\mu}\|_{\infty}
$$

for all discrete measures $\mu$. Now fixing $\mu$ a discrete measure, we have that

$$
\lim _{n \rightarrow \infty}\left|\int f_{n} d \mu\right|=\left|\int \phi d \mu\right|
$$

Hence $\varphi$ satisfies (3), so $\varphi \in A(E)$ by hypothesis, whence

$$
\lim _{n \rightarrow \infty}\left|\left\|f_{n}-\varphi \mid\right\|=0\right. \text {. }
$$

Thus, since $A(E)$ is a Banach space under the weaker norm \|\|$\cdot \|$, we have that $\|\cdot \cdot\|$ is equivalent to the norm $\|\cdot\|_{A(E)}$.

REMARK 1. Walter Rudin has constructed a closed independent set $E$ which supports a measure whose Stieltjes transform vanishes at infinity (see [6]). Such a set does not satisfy the conclusion of Proposition $B$, since the independence of $E$ implies that $\|f\|\|=\| f \|_{\infty}$ for all $f \in A(E)$, whereas the set cannot have its $C(E)$ and $A(E)$ norms equivalent (cf. [5], pp. 114-120). 
REMARK 2. It follows from a theorem of Banach (Theorem 2, p. 213 of [2]), that the conclusion of Proposition $B$ is equivalent to the following: if $F \in A(E)^{*}$, then there exists a sequence of discrete measures $\mu_{n}$ such that $\mu_{n}$ tends to $F$ in the weak * topology of $A(E)$. $\left(A(E)^{*}\right.$ denotes the conjugate space of $A$; the definition of $A(E)$ imples that if $\mu$ is a measure supported on $E$, then $\|\mu\|_{A(E)^{*}}=\|\hat{\mu}\|_{1 \infty}$, where $\|\mu\|_{A(E)^{*}}=\sup \left|\int f d \mu\right|$, the supremum being taken over all $f \in A(E)$ with $\|f\|_{A(E)} \leqq 1$.

In the terminology of [4] (cf. p. 115), our Theorem 3 thus implies that if $E$ is of spectral synthesis and of uniformly positive measure, and if $S$ is a pseudo-measure carried by $E$, there exists a sequence of linear combinations of point masses carried by $E$ and tending weakly to $S$.

We note finally, that Proposition A holds for arbitrary locally compact abelian groups, and Proposition B holds for compact subsets of l.c.a. groups.

2. Throughout this section, $E$ denotes a subset of $\boldsymbol{T}$ of positive Lebesgue measure; $m$ denotes Lebesgue measure on $\boldsymbol{T}$ (with $m(\boldsymbol{T})=1$ ); if $S$ and $T$ are two subsets of $\boldsymbol{T}$,

$$
S+T=\{s+t: s \in S \text { and } t \in T\} .
$$

If $\psi$ is a Lebesgue-integrable function defined on a closed set $E_{1}$, and if $\phi$ is a bounded measurable function defined on a closed set $E_{2}$, we recall that the continuous function $\varphi * \psi$, defined by

$$
(\varphi * \psi)(y)=\int_{0}^{1} \varphi(y-x) \psi(x) d x \quad \text { for all } y \in \boldsymbol{T},
$$

is supported on the set $E_{1}+E_{2}$.

Finally, if $S$ is a subset of $\boldsymbol{T}, \chi_{s}$ denotes the characteristic function of $S$. i.e.

$$
\chi_{s}(y)=1 \text { if } y \in S ; \quad \chi_{s}(y)=0 \quad \text { otherwise. }
$$

Lemma 1. Given $E$ and $\delta>0$, for all sufficiently large integers $N$ there exists a closed subset $F^{\prime} \subset E$, depending on $N$, with $m\left(F^{\prime}\right) \geqq$ $(1-\delta) m(E)$, so that for some $0 \leqq \beta<1 / N$, each of the numbers $\beta+k / N, k=0,1, \cdots, N-1$; either belongs to $F^{\prime}$, or is a distance at least $1 / N$ away from $F^{\prime}$.

Proof. Let $\varepsilon>0$ be given. Then we may choose a closed set $F \subset E$, so that $m(F) \geqq m(E)(1-\varepsilon)$, and so that for all $N$ sufficiently 
large,

$$
m\left(F+\left[-\frac{1}{N}, \frac{1}{N}\right]\right) \leqq m(F)(1+\varepsilon)
$$

(We may accomplish this by simply choosing a finite number of disjoint closed intervals which approximate $E$ closely in measure. Precisely, if $S$ and $T$ are two subsets of $T$, let

$$
S \Delta T=(S \cap \mathscr{C} T) \cup(\mathscr{C} S \cap T) .
$$

First, choose $F_{1}$ a closed subset of $E$, with $m\left(E \Delta F_{1}\right)<(\varepsilon / 2) m(E)$. Next, choose $I_{1}, \cdots, I_{p}$ disjoint closed intervals with

$$
m\left(F_{1} \Delta \bigcup_{j=1}^{p} I_{j}\right)<\frac{\varepsilon^{\prime}}{2} m\left(F_{1}\right),
$$

where $\varepsilon^{\prime}=\min \{\varepsilon, 2 \varepsilon /(2+\varepsilon)\}$. Finally, let

$$
F=\bigcup_{j=1}^{p} I_{j} \cap F_{1} ;
$$

then the desired inequalities hold for all integers $N \geqq(4 p / \varepsilon m(F))$.

Now fix such an $N$; then

$$
m(F)=\sum_{k=1}^{N} m\left(F \cap\left[\frac{k-1}{N}, \frac{k}{N}\right]\right) .
$$

Let $g$ be defined on $[0,1 / N)$ by

$$
g(x)=\frac{1}{N} \sum_{k=0}^{N-1} \chi_{F}\left(x+\frac{k}{N}\right) .
$$

Then

$$
N \int_{0}^{1 / N} g(x) d m(x)=m(F) .
$$

Since $g(x) \geqq 0$ for all $x$, we must have that $g \geqq(1-\varepsilon) m(F)$ on a set of positive measure; thus, we may choose a $\beta, 0 \leqq \beta<(1 / N)$, with

$$
g(\beta) \geqq(1-\varepsilon) m(F) .
$$

Now consider the family of intervals,

$$
I_{k}=\left[\beta+\frac{k}{N}, \beta+\frac{k+1}{N}\right], \quad \text { for } k=0,1, \cdots, N-1 .
$$

We remark that if $f \in F$ belongs to one of these intervals, then the entire interval is contained in the set $F+[-1 / N, 1 / N]$. (Of course, 
$\boldsymbol{T}$ equals the union of these intervals).

Thus, let $\mathscr{K}$ be the subset of $\{0,1, \cdots, N-1\}$ so that $k \in \mathscr{K}$ if and only if $I_{k}$ contains a point of $F$. Then

$$
F \subset \bigcup_{k \in \mathscr{S}} I_{k} \subset F+\left[-\frac{1}{N}, \frac{1}{N}\right] \text {. }
$$

Hence if $r$ is the number of elements in $\mathscr{K}$, we have that

$$
m(F) \leqq \frac{r}{N} \leqq m(F)(1+\varepsilon)
$$

Now, let

$$
\mathscr{J}=\left\{I_{k}: k \in \mathscr{K} \text { and both end points of } I_{k} \text { belong to } F\right\} .
$$

We shall show that $\mathcal{J}$ is nonempty; in fact, letting $l$ be the cardinality of $\mathscr{J}$, we shall show that $l$ is very close to $r$.

First, let

$\mathscr{K}^{\prime}=\{k \in \mathscr{K}: \beta+(k / N) \in F\}$; let $q$ be the cardinality of $\mathscr{K}^{\prime}$ : Then $(q / N)=g(\beta)$.

Now, let

$$
\mathscr{K}^{\prime \prime}=\left\{k \in \mathscr{K}^{\prime}: \beta+\frac{k+1}{N} \notin F\right\},
$$

and let $s$ be the cardinality of $\mathscr{K}^{\prime \prime}$. Noticing that $k \in \mathscr{K}^{\prime \prime}$ if and only if $\beta+(k / N)$ is not a left-hand end point of an interval in $\mathcal{J}$, we thus have that $q-s=l$.

Now to each $k \in \mathscr{K}^{\prime \prime}$ corresponds a unique member of $\mathscr{K} \cap \mathscr{C} \mathscr{K}^{\prime}$, namely the least of the numbers $q \in \mathscr{K}$ such that $q>k$ if there are such numbers; otherwise the least number in $\mathscr{K}$. (Recall that $\beta=$ $\beta+1$, as members of $T$.) Thus

$$
\operatorname{card} \mathscr{K}^{\prime \prime} \leqq \operatorname{card}\left(\mathscr{K} \cap \mathscr{C} \mathscr{K}^{\prime}\right) \text {. }
$$

But

$$
\mathscr{K}^{\prime \prime} \cup\left(\mathscr{K} \cap \mathscr{C} \mathscr{K}^{\prime}\right) \cup\left(\mathscr{K}^{\prime} \cap \mathscr{C} \mathscr{K}^{\prime \prime}\right) \subset \mathscr{K} \text {. }
$$

Hence $s+s+q-s \leqq r$. Thus, $q+s \leqq r$. Hence, since $s=q-l$, we obtain that $r-l \leqq 2(r-q)$. Now, let

$$
F^{\prime}=F \cap \bigcup_{J \in \mathcal{J}^{\mathcal{E}}} J \text {. }
$$

Then $F^{\prime}$ has the property that each number $\beta+(k / N)$ belongs to $F^{\prime}$, or is a distance at least $1 / N$ away from $F^{\prime}$. For if $\beta+(k / N)$ is not an endpoint of an interval $J \in \mathscr{J}$, then $\beta+(k / N)$ is at least distance $1 / N$ away from the nearest point in $\mathscr{J}$. Moreover, $F^{\prime}$ was 
obtained by removing at most $r-l$ intervals from $F^{\prime}$, each of length $1 / N$. Thus, recalling that

$$
\frac{r}{N} \leqq m(F)(1+\varepsilon) \text { and } \frac{q}{N} \geqq m(F)(1-\varepsilon),
$$

we have that

$$
\begin{aligned}
m\left(F^{\prime}\right) \geqq m(F)-\frac{r-l}{N} & \geqq m(F)-2\left(\frac{r-q}{N}\right) \\
& \geqq m(F)[1-2[(1+\varepsilon)-(1-\varepsilon)]] \\
& =m(F)(1-4 \varepsilon) \geqq m(E)(1-4 \varepsilon)(1-\varepsilon) .
\end{aligned}
$$

Thus, given $\delta>0$, we simply choose $\varepsilon$ so that

$$
(1-4 \varepsilon)(1-\varepsilon) \geqq(1-\delta) \text {. }
$$

REmarks. We note incidentally that $l / N$ provides a good approximation to $m(E)$, since

$$
m(E)(1+\varepsilon) \geqq \frac{r}{N} \geqq \frac{l}{N} \geqq m\left(F^{\prime}\right) \geqq m(E)(1-4 \varepsilon)(1-\varepsilon) .
$$

This shows that given $\varepsilon>0$, we may, for all $N$ sufficiently large, give an upper estimate to $m(E)-\varepsilon$ by considering some system of equally spaced intervals of length $1 / N$, then adding up the lengths of all these intervals such that both their endpoints belong to $E$.

The next lemma is directed toward showing that if $\varphi$ is a measurable function satisfying (3), then $\varphi$ also satisfies (3) for a larger class of measures supported on $E$. (See the first line of the proof of Theorem 3.)

LEMMA 2. Let $\varphi$ be a bounded measurable function defined on $E$. Then there exists a sequence of discrete measures $\left\{\nu_{M}\right\}$ supported on $E$, so that

$$
\begin{array}{ll}
\left\|\nu_{M}\right\| \leqq\|\varphi\|_{\infty} & \text { for all } M, \text { with } \\
\left\|\hat{\nu}_{M}\right\|_{\infty} \leqq\left(1+\frac{1}{M}\right)\|\hat{\varphi}\|_{\infty} & \text { and } \\
\lim _{M \rightarrow \infty} \hat{\nu}_{M}(l)=\hat{\varphi}(l) & \text { for all integers } l .
\end{array}
$$

Proof. Fix $M$ an integer. Since $\varphi d m$ is absolutely continuous with respect to $m$, we may choose a $\delta>0$ so that if $K$ is a Lebesgue measurable set with $m(K) \leqq \delta$, then 


$$
\int_{\boldsymbol{K}}|\varphi| d m<\frac{1}{M}\|\hat{\phi}\|_{\infty} .
$$

(Of course we assume that $\|\varphi\|_{1}>0$.) Now by Lemma 1, we may choose a closed set $F \subset E$, an integer $N \geqq M$, and a number $0 \leqq$ $\beta<(1 / N)$, so that $m(E \cap \mathscr{C} F) \leqq \delta$, and so that each of the numbers $\beta+(k / N)$, for $k=0,1, \cdots, N-1$, either belongs to $F$, or is a distance at least $1 / N$ from $F$. Let $\varphi^{\prime}$ be the restriction of $\varphi$ to $F$, i.e. $\varphi^{\prime}=\varphi \chi_{F}$.

Let $m_{N \beta}$ be the discrete measure supported on $\{\beta+(k / N)\}_{k=0}^{N-1}$, and which assigns mass $1 / N$ to each of the points $\beta+k / N$.

Now let $\Delta_{N}$ be the function whose graph is an isosceles triangle of height $N$ and base $[-1 / N, 1 / N]$. Finally, let

$$
\nu_{M}=\left(\Delta_{N} * \varphi^{\prime}\right) m_{N \beta} \text {. }
$$

Now, since $\Delta_{N}^{*} \varphi^{\prime}$ is supported on $F+[-1 / N, 1 / N]$, it follows that $\nu_{M}$ is supported on $F$. Moreover,

$$
\left\|\Delta_{N}\right\|_{1}=1,\left\|\varphi^{\prime}\right\|_{\infty} \leqq\|\varphi\|_{\infty}, \quad \text { and } \quad\left\|m_{N \beta}\right\|=1 ;
$$

hence

$$
\left\|\nu_{M}\right\| \leqq\left\|\Delta_{N} * \varphi^{\prime}\right\|_{\infty}\left\|m_{N \beta}\right\| \leqq\|\varphi\|_{\infty} .
$$

For the next two assertions of the Lemma, we need the following easily established properties of $\hat{\Delta}_{N}$ and $\hat{m}_{N \beta}$ :

(a) $\widehat{\Delta}_{N}(j) \geqq 0$ for all $j$.

(b) $\sum_{l=-\infty}^{\infty} \Delta_{N}(l)=N$.

(c) $\sum_{j=-\infty}^{\infty} \hat{\Delta}_{N}(l+j N)=1$ for all integers $l$.

(d) $\lim _{j \rightarrow \infty} \hat{\Delta}_{j}(l)=1$ for all $l$.

(e) $\hat{m}_{N \beta}(j)=0$ if $j$ is not a multiple of $N$; otherwise, $\hat{m}_{N \beta}(j)=e^{-i 2 \pi \beta j}$.

We thus have, for all integers $l$, that

$$
\begin{aligned}
\hat{\nu}_{M}(l) & =\left[\left(\Delta_{N} * \phi^{\prime}\right) m_{N \beta}\right]^{\wedge}(l) \\
& =\sum_{j=-\infty}^{\infty} \hat{\Delta}_{N}(l-j N) \hat{\varphi}^{\prime}(l-j N) e^{-2 \pi i \beta j N} .
\end{aligned}
$$

Hence,

$$
\left|\hat{\nu}_{M}(l)\right| \leqq \sup _{j}\left|\hat{\varphi}^{\prime}(l-j N)\right| \sum_{j=-\infty}^{\infty}\left|\hat{\Delta}_{N}(l-j N)\right| \leqq\left\|\hat{\varphi}^{\prime}\right\|_{\infty} .
$$

By the first two statements of this proof, we have that

$$
\left\|\varphi-\varphi^{\prime}\right\|_{1}<\frac{1}{M}\|\hat{\varphi}\|_{\infty}
$$


from which it follows that

$$
\left\|\hat{\varphi}^{\prime}\right\|_{\infty} \leqq\left(1+\frac{1}{M}\right)\|\hat{\varphi}\|_{\infty} ;
$$

hence the second assertion follows. Finally, we fix $l$ an integer; then

$$
\begin{aligned}
& \left|\hat{\nu}_{M}(l)-\hat{\varphi}(l)\right| \\
& =\left|\hat{\Delta}_{N}(l) \hat{\varphi}^{\prime}(l)-\hat{\varphi}(l)+\sum_{j \neq 0} \hat{\Delta}_{N}(l-j N) \hat{\varphi}^{\prime}(l-j N) e^{-2 \pi i \beta j N}\right| \\
& \leqq \hat{\Delta}_{N}(l)\left|\hat{\varphi}^{\prime}(l)-\hat{\varphi}(l)\right|+\left(1-\hat{\Delta}_{N}(l)\right)|\hat{\varphi}(l)| \\
& +\sup _{j \neq 0}\left|\hat{\varphi}^{\prime}(l-j N)\right| \sum_{j \neq 0} \hat{\Delta}_{N}(l-j N) \\
& <\frac{1}{M}\|\hat{\varphi}\|_{\infty}+3\|\hat{\varphi}\|_{\infty}\left(1-\hat{\Delta}_{N}(l)\right) \text {. }
\end{aligned}
$$

(The last inequality follows from (c) and the fact that $\left\|\hat{\varphi}^{\prime}\right\|_{\infty} \leqq$ $2\|\hat{\varphi}\|_{\infty}$.) Hence by $(d)$, we have that $\lim _{M \rightarrow \infty} \hat{\nu}_{M}(l)=\hat{\phi}(l)$ for all integers $l$.

Theorem 3. Let $E$ be a closed subset to $\boldsymbol{T}$ of uniformly positive measure. Then if $\psi \in C(E)$ and if $\psi$ satisfies condition (3) with the constant $K$, there exists an $f \in A$ with $\|f\|_{A} \leqq K$, and with $\left.f\right|_{E}=\psi$.

Proof. First, the hypotheses together with Lemma 2 show that

$$
\left|\int \psi \varphi d m\right| \leqq K\|\hat{\varphi}\|_{\infty}
$$

for all bounded measurable functions $\varphi$ supported on $E$.

Indeed, fix such a $\varphi$, and choose $\left\{\nu_{M}\right\}$ a sequence of discrete measures supported on $E$ and satisfying the conclusion of Lemma 2. Since the total variations of the sequence are uniformly bounded, it follows that $\nu_{M}$ tends to $\varphi$ in the weak* topology of $C(E)^{*}$. (Some subsequence converges by Alaoglu's theorem, but any convergent subsequence must converge to $\varphi$ by the uniqueness of Fourier-Stieltjes transforms.) Hence,

$$
\lim _{M \rightarrow \infty} \int \psi d \nu_{M}=\int \varphi \psi d m
$$

Thus,

$$
\left|\int \varphi \psi d m=\lim _{M \rightarrow \infty}\right| \psi d \nu_{M} \mid \leqq \varlimsup_{M \rightarrow \infty} K\left\|\hat{\nu}_{M}\right\|_{\infty} \leqq K\|\hat{\varphi}\|_{\infty} .
$$

Now, let $X$ be the subspace of $c_{0}(\boldsymbol{Z})$, the sequences on the integers vanishing at infinity, defined as 
$X=\{\widehat{\varphi}: \varphi$ is a bounded measurable function, defined on $E\}$. Now define $F$ a linear functional on $X$ by

$$
F(\hat{\varphi})=\int \psi \varphi d m
$$

(Since $\widehat{\varphi}_{1}=\widehat{\varphi}_{2}$ if and only if $\varphi_{1}=\varphi_{2}$ a.e., $F$ is well defined.) Thus $F$ is a bounded linear functional on $X$; so by the Hahn-Banach theorem and the fact that $c_{0}(\boldsymbol{Z})^{*}$ may be identified with $L^{1}(\boldsymbol{Z})$ (the space of all absolutely convergent sequences), there exists an $f \in A$, with $\|f\|_{A} \leqq K$, so that

$$
F(\hat{\varphi})=\sum_{n=-\infty}^{\infty} \hat{\varphi}(n) \hat{f}(-n)=\int f \varphi d m
$$

for all bounded measurable $\varphi$ supported on $E$. The last equality shows that $f=\psi$ a.e.; since $\psi$ is continuous and $E$ is of uniformly positive measure, this implies that $\left.f\right|_{E}=\psi$.

We are finally prepared to establish the analogue of our main result for the circle group $\boldsymbol{T}$.

THEOREM 4. Let $\psi$ be a bounded measurable function defined on $E$, and satisfying (3) with constant $K$. Then there exists an $f \in A$ with $\|f\|_{A} \leqq K$, and such that

$$
f(e)=\psi(e) \text { for almost all } e \in E .
$$

Proof. By Lusin's theorem, given an integer $N$, we may choose $F$ a closed subset of $E$, with $m(E \cap \mathscr{C} F)<(1 / N)$, so that $\psi \mid F$ is continuous; let $\psi_{N}$ denote $\left.\psi\right|_{F}$. We may also assume that $F$ is of uniformly positive measure, by simply taking $N$ large enough and replacing $F$ by the support of the measure $\chi_{F} d m$, if necessary.

For each $N, \psi_{N}$ satisfies the hypotheses of Theorem 3, with constant $K$. Hence we may choose an $f_{N} \in A$, with $\left\|f_{N}\right\|_{A} \leqq K$ and $\left.f_{N}\right|_{F}=\psi_{N}$. Again by Alaoglu's theorem, since the $\hat{f}_{N}$ 's are uniformly bounded in $c_{0}(\boldsymbol{Z})^{*}$, there exists a function $\tau$ defined on $\boldsymbol{Z}$ and a subsequence $\hat{f}_{N_{j}}$ of the $\hat{f}_{N}$ 's, so that

$$
\|\tau\|_{L^{1}(\mathbf{Z})}=\sum_{n=-\infty}^{\infty}|\tau(n)| \leqq K
$$

and so that

$$
\lim _{j \leftarrow \infty} \sum_{n=-\infty}^{\infty} \hat{f}_{N_{j}}(n) \beta(-n)=\sum_{n=-\infty}^{\infty} \tau(n) \beta(-n)
$$

for all $\beta \in c_{0}(Z)$. Thus, let 


$$
f(x)=\sum_{-\infty}^{\infty} \tau(n) e^{2 \pi i n x}
$$

for all $x \in T$; then $\|f\|_{A} \leqq K$, and

$$
\lim _{j \rightarrow \infty} \int f_{N_{j}} \varphi d m=\int f \varphi d m
$$

for all bounded measurable functions $\varphi$ defined on $E$. But fix such a $\varphi$; then

$$
\lim _{N \rightarrow \infty} \int f_{N} \varphi d m=\int \psi \varphi d m
$$

indeed, for fixed $N$, taking the corresponding $F$ as in the first statement of this proof, we have that

$$
\int\left|f_{N}-\psi\right| \varphi d m=\int_{E \cap \mathscr{E} F}\left|f_{N}-\psi\right| \varphi d m \leqq \frac{1}{N}\left(K+\|\psi\|_{\infty}\right)\|\varphi\|_{\infty} \cdot
$$

Hence, $\psi=f$ a.e. an $E$.

3. Proof of the main result. We first have need of the following lemma, showing that the Stieltjes transform of a finite compactly supported measure on the real line may be nicely approximated by its values on a discrete subset.

Lemma 5. Given $\varepsilon$ and $N>0$, there exists an $M>0$, so that if $L \geqq M$ and if $\nu$ is a finite measure supported on $[-N, N]$,

$$
\sup _{x \in R}|\hat{\nu}(x)| \leqq(1+\varepsilon) \sup _{j \in Z}\left|\hat{\nu}\left(\frac{\pi j}{L}\right)\right| .
$$

Proof. We first note that given $\lambda$ real number, there exists $f \in L^{1}(\boldsymbol{R})$ with $\hat{f}(x)=e^{i \lambda x}-1$ for all $|x| \leqq N$, and such that $\|f\|_{1} \leqq$ $6|\lambda| N$. For example, let

$$
k(x)=\frac{1}{2 N}\left(\chi_{[-N, N]}\right)^{\wedge}(x)\left(\chi_{[-2 N, 2 N]}\right)^{\wedge}(x)
$$

for all real $x$, and set

$$
f(x)=\frac{1}{2 \pi}(k(x+\lambda)-k(x))
$$

for all real $x$.

(To see that $f$ has the desired properties, one may use an argument analogous to that given in the proof of 2.6.3, page 49 of [5]. Briefly, for $|y| \leqq N$, we have that 


$$
\begin{aligned}
& \frac{1}{2 N} \chi_{[-N, N] * \chi_{[-2 N, 2 N]}}(y)=1 ; \text { hence } \\
& \hat{f}(y)=\left(e^{i \lambda y}-1\right) \frac{\hat{k}(y)}{2 \pi}=e^{i \lambda y}-1
\end{aligned}
$$

by the inversion theorem. Now

$$
\begin{aligned}
& f(x)=\frac{1}{2 \pi} \frac{1}{2 N}\left(e^{i \lambda \cdot} \chi_{[-N, N]}\right)^{\wedge}(x)\left(\left(e^{i \lambda \cdot}-1\right) \chi_{[-2 N, 2 N]}\right)^{\wedge}(x) \\
& +\frac{1}{2 \pi} \frac{1}{2 N}\left(\left(e^{i \lambda \cdot}-1\right) \chi_{[-N, N]}\right)^{\wedge}(x)\left(\chi_{[-2 N, 2 N]}\right)^{\wedge}(x) .
\end{aligned}
$$

Hence by the Plancherel theorem and the Schwartz inequality,

$$
\begin{aligned}
& \|f\|_{1} \leqq \frac{1}{2 N}\left\|\chi_{[-N, N]}\right\|_{2} \sup _{|y| \leqq 2 N}\left|e^{i \lambda y}-1\right|\left\|\chi_{[-2 N, 2 N]}\right\|_{2} \\
& \quad+\frac{1}{2 N} \sup _{|y| \leqq N}\left|e^{i \lambda y}-1\right|\left\|\chi_{[-N, N]}\right\|_{2}\left\|\chi_{[-2 N, 2 N]}\right\|_{2} \\
& \quad \leqq 3 \sqrt{2}|\lambda| N ;
\end{aligned}
$$

thus the constant " 6 " could be replaced by the constant " $3 \sqrt{2} \overline{2}$ ".)

Now, suppose $L>6 \pi N, \nu$ is supported on $[-N, N]$, and fix $x$ a real number. Let $j$ be the integer such that

$$
\frac{\pi j}{L} \leqq x<\frac{\pi(j+1)}{L}
$$

Next, choose $f$ as in the first statement of the proof, with $\lambda=(\pi j / L)-x$, and let $f_{1}(y)=f(y-(\pi j / L))$ for all real $y$. Then

$$
\begin{aligned}
& \left|\hat{\nu}(x)-\hat{\nu}\left(\frac{\pi j}{L}\right)\right| \\
& \quad=\left|\int_{-N}^{N}\left(e^{-i x t}-e^{-i(\pi j / L) t}\right) d \nu(t)\right| \\
& =\left|\int_{-\infty}^{\infty} \hat{f}_{1}(t) d \nu(t)\right| \\
& =\left|\int_{-\infty}^{\infty} \hat{\nu}(t) f_{1}(t) d t\right| \\
& \quad \leqq\|\hat{\nu}\|_{\infty}\left\|f_{1}\right\|_{1} \leqq 6|\lambda| N\|\hat{\nu}\|_{\infty} \leqq 6 N \frac{\pi}{L}\|\hat{\nu}\|_{\infty} .
\end{aligned}
$$

Hence,

$$
|\hat{\nu}(x)|-6 N \frac{\pi}{L}\|\hat{\nu}\|_{\infty} \leqq\left|\hat{\nu}\left(\frac{\pi j}{L}\right)\right| \leqq \sup _{k \in Z}\left|\hat{\nu}\left(\frac{\pi k}{L}\right)\right|
$$


Thus, since $x$ was arbitrary,

$$
\|\hat{\nu}\|_{\infty} \leqq \frac{1}{1-\frac{6 N \pi}{L}} \sup _{k \in Z}\left|\hat{\nu}\left(\frac{\pi k}{L}\right)\right| .
$$

So, given $\varepsilon>0$, simply choose $M$ so that $L \geqq M$ implies that

$$
\frac{1}{1-\frac{6 N \pi}{L}} \leqq 1+\varepsilon .
$$

REMARK. Our proof shows that the conclusion of Lemma 5 holds not only for Stieltjes transforms, but for any bounded continuous function $\varphi$ whose spectrum is supported on the interval $[-N, N]$, i.e. we obtain that

$$
\sup |\varphi(x)| \leqq(1+\varepsilon) \sup _{j \in Z}\left|\varphi\left(\frac{\pi j}{L}\right)\right|
$$

for all $L \geqq M$.

Proof of the main result. (All terms are as defined on the first page of this paper.)

Fix $N$ an integer; by Lemma 5 , we may choose $L>N$ so that if $\nu$ is a finite measure supported on $[-N, N]$, then

$$
\sup _{x \in R}|\hat{\nu}(x)| \leqq\left(1+\frac{1}{N}\right) \sup _{j \in Z}\left|\hat{\nu}\left(\frac{\pi j}{L}\right)\right| \text {. }
$$

We assume that $\varphi$ satisfies condition (1), or equivalently, condition (3); let $\varphi_{N}=\left.\varphi\right|_{E \cap[-N, N]}$. $\varphi_{N}$ may be considered as being defined on a closed subset of the reals modulo $2 L$; we then have that if $\nu$ is a discrete measure supported on $E \cap[-N, N]$

$$
\left|\int \varphi d \nu\right| \leqq K \sup _{x \in \boldsymbol{R}}|\hat{\nu}(x)| \leqq K\left(1+\frac{1}{N}\right) \sup _{j \in Z}\left|\hat{\nu}\left(\frac{\pi}{L} j\right)\right| .
$$

Applying the obvious version of Theorem 4 for the reals modulo $2 L$ instead of the reals modulo 1 , we obtain that there exists a sequence $\left\{a_{j}\right\}$ with

$$
\sum_{j=-\infty}^{\infty}\left|a_{j}\right|<\left(1+\frac{1}{N}\right) K
$$

such that

$$
\varphi_{N}(x)=\sum a_{j} e^{i(\pi / L) j x}
$$


for almost all $x \in E \cap[-N, N]$.

Now let $\mu_{N}$ be the discrete measure which, for each integer $j$, assigns mass $a_{-j}$ to the point $(\pi / L) j$; then $\varphi_{N}=\hat{\mu}_{N}$ a.e. on $E \cap[-N, N]$, and $\left\|\mu_{N}\right\| \leqq(1+(1 / N)) K$.

Finally, by Alaoglu's theorem, since the finite measures on $\boldsymbol{R}$ may be identified with the adjoint of $C_{0}(\boldsymbol{R})$, the Banach space of continuous functions vanishing at infinity, we may choose a finite measure $\mu$, with $\|\mu\| \leqq K$, and a subsequence $\left\{\mu_{N_{j}}\right\}$ so that

$$
\int f d \mu=\lim _{j \rightarrow \infty} \int f d \mu_{N_{j}}
$$

for all $f \in C_{0}(\boldsymbol{R})$. Now if $g$ is a continuous function with compact support, then

$$
\begin{aligned}
& \int_{-\infty}^{\infty} g(x) \varphi(x) d x \\
& \quad=\lim _{j \rightarrow \infty} \int_{-\infty}^{\infty} g(x) \varphi_{N_{j}}(x) d x \\
& \quad=\lim _{j \rightarrow \infty} \int_{-\infty}^{\infty} g(x) \hat{\mu}_{N_{j}}(x) d x \\
& \quad=\lim _{j \rightarrow \infty} \int_{-\infty}^{\infty} \hat{g}(x) d \mu_{N_{j}}(x)=\int_{-\infty}^{\infty} \hat{g}(x) d \mu(x)=\int_{-\infty}^{\infty} g(x) \hat{\mu}(x) d x .
\end{aligned}
$$

Hence $\hat{\mu}=\varphi$ a.e.

REMARK. For the sake of simplicity in notation, we have only considered the one-dimensional case. However, all our results also hold in the context of $\boldsymbol{R}^{p}$ and $\boldsymbol{T}^{p}$ for all $p>1$. We indicate briefly the necessary changes in the notation and arguments.

We identify $\boldsymbol{T}^{p}$ with $\boldsymbol{R}^{p} / \boldsymbol{Z}^{p}$, and endow both $\boldsymbol{T}^{p}$ and $\boldsymbol{R}^{p}$ with the sup of coordinates metric. If $a$ and $b$ are real numbers, we define the half-open $p$-dimensional interval

$$
[a, b)_{p}=\left\{x \in \boldsymbol{R}^{p}: x=\left(x_{1}, \cdots, x_{p}\right) \text { and } a \leqq x_{j}<b \text { for all } 1 \leqq j \leqq p\right\} \text {. }
$$

Similarly, we define closed and open intervals. If $x \in \boldsymbol{R}^{p}$ and $n \in \boldsymbol{Z}^{p}$, we define

$$
x n=n x=n_{1} x_{1}+\cdots+n_{p} x_{p} .
$$

We then replace " $\boldsymbol{Z}$ ", " $\boldsymbol{R}$ " and " $\boldsymbol{T}$ " by " $\boldsymbol{Z}$ ", " $\boldsymbol{R}^{p}$ ", and " $\boldsymbol{T}$ " respectively, throughout the paper. Where summation indices run over $\boldsymbol{Z}$, we thus allow them to run over $\boldsymbol{Z}^{p}$, and where integrals are taken over intervals, we take them over $p$-dimensional intervals. With these changes, the statements and proofs of Theorems 3, 4, and the main result are exactly the same; a few more modifications are 
required for the proofs of the three lemmas, as follows:

In Lemmas 1 and 2 , we take $\beta$ to be a point in $[0,1 / N)_{p}$. In the proof of Lemma 1 , we allow the indices " $k$ " to range over all $k \in \boldsymbol{Z}^{p}$ such that $k=\left(k_{1}, \cdots, k_{p}\right)$ and $0 \leqq k_{j} \leqq N-1$ for all $1 \leqq j \leqq p$. For each such $k$, we define

$$
I_{k}=\beta+\frac{k}{N}+\left[0, \frac{1}{N}\right]_{p} .
$$

$\mathcal{J}$ is then defined to be all intervals such that all of their endpoints belong to $F$; i.e.

$$
\begin{aligned}
& \mathscr{J}=\left\{I_{k} \text { : for all } x \in Z^{p} \text { such that } x_{j}=0 \text { or } 1 \text { for all } j\right. \\
& \text { we have that } \left.\beta+\frac{k+x}{N} \in F\right\} .
\end{aligned}
$$

Exactly the same definitions are given for $\mathscr{K}$ and $\mathscr{K}^{\prime}$, then $\mathscr{K}^{\prime \prime}$ is defined as

$$
\begin{aligned}
& \left\{k \in \mathscr{K}^{\prime}: \text { there exists an } x \in \boldsymbol{Z}^{p} \text { with } x_{j}=0 \text { or } 1 \text { all } j,\right. \\
& \text { so that } \left.\beta+\frac{k+x}{N} \notin F\right\} .
\end{aligned}
$$

We may then correspond to each member of $\mathscr{K}^{\prime \prime}$ a member of $\mathscr{K} \cap \mathscr{C} \mathscr{K}^{\prime}$ as follows:

Given $k \in \mathscr{K}^{\prime \prime}$, choose $x \in \boldsymbol{Z}^{p}$ with $x_{j}=0$ or 1 for all $j$, such that $\beta+((k+x) / N) \notin F$. Now let $l$ be the least integer with $1 \leqq l \leqq N-1$ so that there exists a $q \in \mathscr{K}$ and an $m \in \boldsymbol{Z}^{p}$ with $k+l x-q=N m \quad$ (i. e. $\quad$ such that $k+l x \equiv q \bmod N \boldsymbol{Z}^{p}$ ); then $q \in \mathscr{K} \cap \mathscr{C} \mathscr{K}^{\prime}$, so we correspond $q$ to $k$.

Given such a $q$ and such an $x, k$ is uniquely determined by the relation $k \equiv q-l x \bmod N Z^{p}$, where $l$ is chosen to be the least integer with $1 \leqq l \leqq N-1$, so that $\beta+((q-l x) / N) \in F$.

However, for different $x$ 's, we may have different $k$ 's in $\mathscr{K}^{\prime \prime}$ corresponded to the same $q$ in $\mathscr{K} \cap \mathscr{C} \mathscr{C}^{\prime \prime}$. Since there are at most $2^{p}-1$ such $x$ 's $\left(x_{j}\right.$ must equal 1 for some $j$ ), it follows that

$$
\frac{1}{2^{p}-1} \operatorname{card} \mathscr{K}^{\prime \prime} \leqq \operatorname{card}\left(\mathscr{K} \cap \mathscr{C} \mathscr{K}^{\prime}\right) \text {. }
$$

We thus obtain that $r-l \leqq 2^{p}(r-q)$, where $r, l$, and $q$ are as defined in Lemma 1; the term " $4 \varepsilon$ " must then be replaced by the term " $2^{p+1} \varepsilon$ ".

One other modification is required: in all rational numbers having $N$ as denominator (and not having a " $k$ " as a numerator!), we replace " $N$ " by " $N$ "'. Thus the function $g(x)$ is defined on $[0,1 / N)$ ), by 


$$
g(x)=\frac{1}{N^{p}} \sum_{\substack{k, j=0 \\ 1 \leqq j \leqq p}}^{N-1} \chi_{F}\left(x+\frac{k}{N}\right)
$$

we then have that

$$
N^{p} \int_{[0,1 / N)_{p}} g d m=m(F) .
$$

For the proof of Lemma 2, we replace the function $\Delta_{N}$ by the function

$$
\Delta_{N, p}=N^{2 p} \chi_{[0,1 / N]_{p}} * \chi_{[-1 / N, 0]_{p}} \cdot
$$

$m_{N \beta}$ is then defined as the discrete measure which assigns mass $1 / N^{p}$ to each of the points $\beta+(k / N)$, where $k=\left(k_{1}, \cdots, k_{p}\right)$ and $0 \leqq k_{q} \leqq$ $N-1$ for all $1 \leqq q \leqq p$. Exactly the same proof then holds.

Finally, in the proof of Lemma 5, the number " 6 " should be replaced by a constant $K$ that depends only on $p$. (Of course, $\lambda$ is taken as a point in $\boldsymbol{R}^{p}$, with $\left.|\lambda|=\sup _{1 \leq j \leq p}\left|\lambda_{j}\right|.\right)$ An example of a function with the property given in the first line of the proof of Lemma 5, may then be obtained by setting

$$
k(x)=\frac{1}{2^{p} N^{p}}\left(\chi_{[-N, N]_{p}}\right)^{\wedge}(x)\left(\chi_{[-2 N, 2 N]}\right)^{\wedge}(x)
$$

for all $x \in \boldsymbol{R}^{p}$, and then putting

$$
f(x)=\frac{1}{2^{p} \pi^{p}}(k(x+\lambda)-k(x)) \text { for all } x \in \boldsymbol{R}^{p} .
$$

\section{REFERENCES}

1. N. T. Akhiezer, Theory of Approximation, Ungar Publ. Co., New York, 1956.

2. S. Banach, Theorie des Operations Lineaires, Monografje Matematyczne, Warsaw, 1932.

3. S. Bochner, A theorem on Fourier-Stieltjes integrals, Bull. Amer. Math. Soc. 40 (1934), 271-276.

4. J. P. Kahane and R. Salem, Ensembles Parfaits et séries Trigonometriques, Hermann, Paris, 1963.

5. W. Rudin, Fourier Analysis on Groups, Interscience Publ., New York, 1962.

6. - Fourier-Stieltjes transforms of measures on independent sets, Bull. Amer. Math. Soc. 66 (1960), 199-202.

Received January 17, 1967. This research was partially supported by NSF-GP-5585.

UNIVERSity of CALifornia AT Berkeley 


\section{PACIFIC JOURNAL OF MATHEMATICS}

\section{EDITORS}

\section{H. ROYDEN \\ Stanford University \\ Stanford, California}

\author{
J. P. JANS \\ University of Washington \\ Seattle, Washington 98105
}

J. DugundJI

Department of Mathematics

Rice University

Houston, Texas 77001

RichaRd ARENS

University of California

Los Angeles, California 90024

\section{ASSOCIATE EDITORS}
E. F. BECKENBACH
B. H. NeumanN
F. WOLF
K. YOSIDA

\section{SUPPORTING INSTITUTIONS}

\author{
UNIVERSITY OF BRITISH COLUMBIA \\ CALIFORNIA INSTITUTE OF TECHNOLOGY \\ UNIVERSITY OF CALIFORNIA \\ MONTANA STATE UNIVERSITY \\ UNIVERSITY OF NEVADA \\ NEW MEXICO STATE UNIVERSITY \\ OREGON STATE UNIVERSITY \\ UNIVERSITY OF OREGON \\ OSAKA UNIVERSITY \\ UNIVERSITY OF SOUTHERN CALIFORNIA
}

\author{
STANFORD UNIVERSITY \\ UNIVERSITY OF TOKYO \\ UNIVERSITY OF UTAH \\ WASHINGTON STATE UNIVERSITY \\ UNIVERSITY OF WASHINGTON

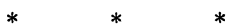 \\ AMERICAN MATHEMATICAL SOCIETY \\ CHEVRON RESEARCH CORPORATION \\ TRW SYSTEMS \\ NAVAL ORDNANCE TEST STATION
}

Mathematical papers intended for publication in the Pacific Journal of Mathematics should be typewritten (double spaced). The first paragraph or two must be capable of being used separately as a synopsis of the entire paper. It should not contain references to the bibliography. Manuscripts may be sent to any one of the four editors. All other communications to the editors should be addressed to the managing editor, Richard Arens at the University of California, Los Angeles, California 90024.

50 reprints per author of each article are furnished free of charge; additional copies may be obtained at cost in multiples of 50 .

The Pacific Journal of Mathematics is published monthly. Effective with Volume 16 the price per volume (3 numbers) is $\$ 8.00$; single issues, $\$ 3.00$. Special price for current issues to individual faculty members of supporting institutions and to individual members of the American Mathematical Society: $\$ 4.00$ per volume; single issues $\$ 1.50$. Back numbers are available.

Subscriptions, orders for back numbers, and changes of address should be sent to Pacific Journal of Mathematics, 103 Highland Boulevard, Berkeley 8, California.

Printed at Kokusai Bunken Insatsusha (International Academic Printing Co., Ltd.), 7-17, Fujimi 2-chome, Chiyoda-ku, Tokyo, Japan.

PUBLISHED BY PACIFIC JOURNAL OF MATHEMATICS, A NON-PROFIT CORPORATION

The Supporting Institutions listed above contribute to the cost of publication of this Journal, but they are not owners or publishers and have no responsibility for its content or policies. 


\section{Pacific Journal of Mathematics}

\section{Vol. 23, No. 2 \\ April, 1967}

Herbert Stanley Bear, Jr. and Bertram John Walsh, Integral kernel for

one-part function spaces .......................... 209

Mario Borelli, Some results on ampleness and divisorial schemes ....... 217

John A. Erdos, Unitary invariants for nests . ................... 229

Nathaniel Grossman, The volume of a totally-geodesic hypersurface in a pinched manifold.................................. 257

D. M. Hyman, A generalization of the Borsuk-Whitehead-Hanner theorem ............................................. 263

I. Martin (Irving) Isaacs, Finite groups with small character degrees and large prime divisors ............................. 273

I. Martin (Irving) Isaacs, Two solvability theorems ................ 281

William Lee Johnson, The characteristic function of a harmonic function in a locally Euclidean space ............................... 291

Ralph David Kopperman, Application of infinitary languages to metric spaces ............................................. 299

John Lauchlin MacDonald, Relative functor representability ............ 311

Mahendra Ganpatrao Nadkarni, A class of measures on the Bohr group.... 321

Keith Lowell Phillips, Hilbert transforms for the p-adic and p-series fields....................................... 329

Norman R. Reilly and Herman Edward Scheiblich, Congruences on regular semigroups ...

Neil William Rickert, Measures whose range is a ball ... . .

Gideon Schwarz, Variations on vector measures

Ronald Cameron Riddell, Spectral concentration for self-adjoint operators. .

Haskell Paul Rosenthal, A characterization of restrictions of

Fourier-Stieltjes transforms ................... 